\title{
LaserFactory: A Laser Cutter-based Electromechanical Assembly and Fabrication Platform to Make Functional Devices \& Robots
}

\author{
Martin Nisser \\ MIT CSAIL \\ Cambridge, Massachusetts \\ nisser@mit.edu \\ Aradhana Adhikari \\ MIT CSAIL \\ Cambridge, Massachusetts \\ adhikara@mit.edu
}

\author{
Christina Liao \\ MIT CSAIL
}

Cambridge, Massachusetts

ccliao@mit.edu

Steve Hodges

Microsoft Research Cambridge, UK

Cambridge, United Kingdom

steve.hodges@microsoft.com

\author{
YuChen Chai \\ MIT CSAIL
}

Cambridge, Massachusetts

ycchai@mit.edu

Stefanie Mueller

MIT CSAIL

Cambridge, Massachusetts

stefanie.mueller@mit.edu
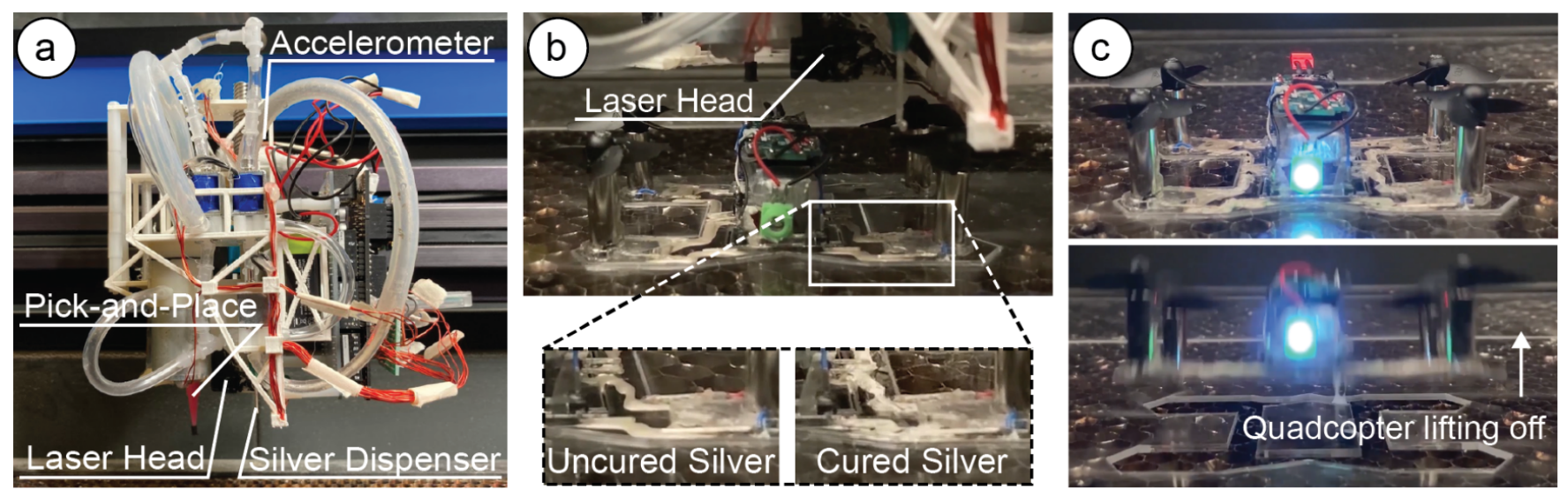

Figure 1: LaserFactory is an integrated fabrication process that creates fully functional devices. (a) Our hardware add-on to an existing laser cutter consists of a silver dispenser and pick-and-place mechanism and allows the machine to not only cut geometry, but also create circuit traces and assemble electronic components. Our accelerometer-based motion classifier enables the add-on to interface with the laser cutter without the need to change the underlying firmware. (b) To cure the deposited silver traces, we developed a laser soldering method that uses the heat of the defocused laser to make the traces conductive. (c) After laser soldering, the fabricated device is fully functional.

\begin{abstract}
LaserFactory is an integrated fabrication process that augments a commercially available fabrication machine to support the manufacture of fully functioning devices without human intervention. In addition to creating 2D and 3D mechanical structures, LaserFactory creates conductive circuit traces with arbitrary geometries, picks-and-places electronic and electromechanical components, and solders them in place. To enable this functionality, we make four contributions. First, we build a hardware add-on to the laser cutter head that can deposit silver circuit traces and assemble components. Second, we develop a new method to cure dispensed silver using a $\mathrm{CO}_{2}$ laser. Third, we build a motion-based signaling method
\end{abstract}

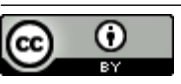

This work is licensed under a Creative Commons Attribution International 4.0 License.

CHI '21, May 8-13, 2021, Yokohama, Japan

(C) 2021 Copyright held by the owner/author(s)

ACM ISBN 978-1-4503-8096-6/21/05.

https://doi.org/10.1145/3411764.3445692 that allows our system to be readily integrated with commercial laser cutters. Finally, we provide a design and visualization tool for making functional devices with LaserFactory. Having described the LaserFactory system, we demonstrate how it is used to fabricate devices such as a fully functioning quadcopter and a sensor-equipped wristband. Our evaluation shows that LaserFactory can assemble a variety of differently sized components (up to $65 \mathrm{~g}$ ), that these can be connected by narrow traces (down to $0.75 \mathrm{~mm}$ ) that become highly conductive after laser soldering $(3.2 \Omega / \mathrm{m})$, and that our accelerationbased sensing scheme works reliably (to $99.5 \%$ accuracy).

\section{CCS CONCEPTS}

- Human-centered computing $\rightarrow$ Human computer interaction (HCI).

\section{KEYWORDS}

Human-computer interaction, rapid prototyping, personal fabrication, printed electronics, robotics 
ACM Reference Format:

Martin Nisser, Christina Liao, YuChen Chai, Aradhana Adhikari, Steve Hodges, and Stefanie Mueller. 2021. LaserFactory: A Laser Cutter-based Electromechanical Assembly and Fabrication Platform to Make Functional Devices \& Robots. In CHI Conference on Human Factors in Computing Systems (CHI '21), May 8-13, 2021, Yokohama, Japan. ACM, New York, NY, USA, 15 pages. https://doi.org/10.1145/3411764.3445692

\section{INTRODUCTION}

To fabricate a fully functional device-a 3D structure with integrated electronics and actuators-requires three steps; (1) fabricating the device geometry, (2) creating conductive circuit traces, and (3) populating the structure with electronic components. Researchers have in recent decades developed numerous personal fabrication methods that democratize the creation of such functional devices. These methods focus on extending the abilities of existing fabrication machines designed to create geometry to also permit creating conductive traces, for example by generating hollow internal pipes for 3D prints that can be filled with conductive material [20], or using laser cutters to selectively ablate conductive films that can be populated with components [5]. However as yet, personal fabrication machines do not have the ability to integrate all three steps, and instead rely on human intervention to complete the fabrication procedure. As such, the fabrication of functional devices continues to demand engineering expertise, for example, to etch PCBs or solder components. In addition, repeatability and precision in the construction is compromised, as techniques based on manual intervention impede the ability for engineered devices to be made to specification.

In this paper, we build upon the above systems by proposing a fabrication machine that creates device geometry, adds circuit traces, and then incorporates electronic components in an automated way. With such a machine, users only need to load components into a storage area at the beginning of the fabrication process and execute the fabrication file in order to create a fully functional device. Since many devices can be created from circuits assembled from a subset of basic electronic parts such as transistors, resistors and capacitors, loading a set of these allows users to download and print a variety of different circuits such as H-bridges and op-amps in order to build devices capable of more complex tasks such as motor driving, display rendering and signal conditioning.

There are two possible pathways to create a fabrication platform that can fabricate geometry, circuit traces, and assemble electronic components: either augmenting an existing fabrication device with a custom hardware add-on or building a new fabrication device from the ground up. The benefit of augmenting an existing fabrication device is that existing infrastructure can be leveraged and thus the engineering effort required to create the functionality can be potentially reduced. In addition, the new fabrication capabilities can be made available to a wide range of users who already own such devices and are familiar with them. However, augmenting existing devices comes with its own challenges since the proprietary software running commercial fabrication devices typically does not allow for modifications, which are necessary to instruct a custom hardware add-on to perform its function. To solve this issue, we developed a technique that allows a hardware add-on to communicate with the commercial fabrication device without the need to change the underlying code. The key idea is to embed signals into the fabrication file that result in specific movements of the fabrication head, which can be sensed with a motion-sensor (accelerometer) attached to the head (Figure 1a). Such signals can inform the hardware add-on about which parts of the fabrication file the fabrication head is currently executing and thus instruct the hardware add-on to perform its function at the right time.

While the motion-based signaling technique for augmenting existing fabrication devices works for different types of fabrication machines granted they use a fabrication head on a motion platform and allow custom motion lines to be written to the fabrication file, our platform centers on a commercial laser cutter. We chose a laser cutter as the fabrication platform due to its speed of operation and thus quick turn-around time for prototyping. To augment the laser cutter with the ability to create circuit traces and assemble electronic components, we developed a custom hardware add-on consisting of a silver dispenser and a pick-and-place mechanism that is mounted onto the existing laser cutter head (Figure 1a). When the embedded motion lines in the fabrication file indicate that a circuit trace needs to be fabricated, the silver dispenser starts laying down silver while the laser's motion carriage continues its movement along the path encoded in the design. Similarly, when the embedded motion lines in the fabrication file indicate that a component needs to picked and placed, a pump connected to the pick-and-place mechanism creates negative pressure for picking up the component.

Since the silver is not yet conductive after it has been dispensed, we developed a technique that uses the heat of the laser for thermal curing of the dispensed silver (Figure 1b). This process is also used to rigidly connect the electronic components to the circuit traces, resulting in the final assembly of the functional device. In contrast to methods that rely on carbon-based conductors and 3D printed filament (Capricate [22]), dispensing and curing the silver creates highly conductive circuit traces. In addition, the laser soldering step also removes the need for a manual curing procedure as was needed in prior work (Valentine et al. [29]). Lastly, in contrast to conductive pastes used in 3D printing-based systems such as Voxel8 [31] that cure uniformly in a specified time and therefore precludes local control, laser soldering selectively cures silver paste when required once components are placed. Thus laser soldering does not rely on paste properties that must be synthesized to trade off slow curing (to allow placing components while paste remains uncured) against fast curing (to expedite fabrication).

To support the design of fully functional devices that can be fabricated on our augmented fabrication machine, we also provide a software toolkit that allows users to design devices in a 2D editor by concurrently creating both their geometry and electronic layout. A custom visualization tool then provides a $3 \mathrm{D}$ rendering of the resulting geometry and displays the order of fabrication steps, which users can use to validate the design before making it. On export, the software then translates the user's custom design into machine instructions embedded in a single fabrication file (.pdf) that can be fabricated using the standard laser cutter software to make the functional device in one go (Figure 1c).

In summary, this paper makes the following contributions: 
- a hardware add-on consisting of a silver dispenser and a pickand-place mechanism to augment an existing laser cutter's functionality with the ability to create circuit traces and place components;

- laser soldering, a technique for curing dispensed silver traces using the heat of the existing $\mathrm{CO}_{2}$ laser to create highly conductive circuit traces;

- a motion-based signaling technique for augmenting an existing fabrication platform without the need to interface with its underlying software;

- a software toolkit consisting of three elements: a tool that supports the concurrent design of device geometry, circuit traces and electronic component layouts; a tool that visualizes the resulting 3D geometry and steps of the fabrication process; and a translation pipeline that converts the design into machine instructions for the fabrication device and hardware add-on.

In the remainder of this paper, we first review the related work and then discuss each of the contributions in order. We then present a technical evaluation of our work and showcase a range of application scenarios.

\section{RELATED WORK}

Our work is related to research that augments existing fabrication machines as well as research that investigates how to make functional electromechanical devices by fabricating circuit traces and assembling electronic components.

\subsection{Fabricating Circuit Traces to Support Electromechanical Devices}

Researchers have developed a variety of methods in pursuit of functional device fabrication. One of the first approaches was to augment passive $3 \mathrm{D}$ printed objects with circuit functionality by adding circuit traces to them after fabrication. For instance, in the Un-Toolkit [13], users sketch circuits using a 2D drawing pen loaded with silver ink. Similarly, in Midas [21], users attach conductive tape to existing objects to enhance them with circuit functionality. Extending this work, robotics researchers printed joule-heating circuits onto copper-clad kapton which were etched in ferric chloride and attached to shape memory polymers to stimulate self-folding of the underlying structure [16]. PipeDream [20] 3D prints objects with internal pipes and then asks users to fill the pipes with copper paint after fabrication to make them conductive. Similarly, Curveboards [35] first 3D prints a housing before requiring channels to be manually filled with conductive silicone. Extending this work, SurfCuit [28] creates channels on the surface of a 3D printed object that users affix copper tape to and solder the components on.

More recently, researchers have investigated how to automate the circuit creation process. For instance, a laminate sheet (base material and conductive copper layer) can be used in a laser cutter to create circuit traces on the sheet's surface via cutting (Foldtronics [34]) or ablation (LASEC [5]). Another technology, inkjet printing of silver ink, can print circuit traces directly onto existing 2D substrates (Instant Inkjet Circuits [8], Building Functional Circuits [9]). With the availability of conductive filament for 3D printing, researchers have also started to co-create object geometry and circuit traces in one integrated process. PrintPut [2], Capricate [22], ./Trilaterate [24], and Flexibles [23] all use carbon-based conductive filament alongside regular filament to fabricate capacitive touch sensors and wires onto 3D geometries. Carbon-based conductive filament, however, has a high trace resistance and does not support all forms of electronics. Newer approaches such as 3D printing with silver ink (Voxel8 [31]) and carbon fiber (FiberWire [25]) produce lower resistance connections and therefore increase the range of electronic components and circuits that are practical.

\subsection{Automatically Integrating Electronic Components}

None of the techniques mentioned above automatically integrate electronic components as part of the fabrication process, which is a task that must be completed after fabrication, usually manually. Hodges et al. demonstrate how to facilitate this process by attaching electronic components to stickers, which can then be added to the circuit (Circuit Stickers [6]). An approach by Valentine et al. [29] uses a silver dispenser to create circuit traces and a pickand-place mechanism to automatically assemble components onto a commercial 3-axis motion control stage. However, without adding a dedicated curing mechanism to their additive direct-writing fabrication process, it lacks the capability to cure the traces during fabrication. Thus, following fabrication, the resulting devices from Valentine et al. must be manually removed and separately cured at elevated temperatures in an oven for an additional two hours to be made functional. This can cause problems as existing work has shown that when electronic components are placed into an oven to cure silver, the temperature can damage the parts (Shrinky Circuits [12]). In addition, subjecting the entire material substrate to high temperature may cause warping or degradation of many additively printed polymers. To address this issue, we build our hardware augmentation onto an existing laser cutter and use the heat of the laser to both cure traces made from silver paste and also solder components into place. For this, we build on work from Lambrichts et al. [10], who used a laser to cure solder paste. Solder paste is different from silver paste in that it can be used to solder components but not to create circuit traces as it beads up when soldered due to surface tension. Researchers have previously used laser sintering of inkjet-printed silver nanoparticle inks to fabricate traces on paper substrates, however these traces were pre-baked in an oven at $110^{\circ} \mathrm{C}$ for 20 minutes, and required components to be added separately via traditional soldering [1]. In contrast, we demonstrate that laser soldering can be used to both cure silver paste without pre-baking the silver compound, and to simultaneously bond components, yielding a method that can quickly create and cure circuit traces and thus can make fully functional devices in just a few minutes. As laser soldering addresses specific traces selectively, it also removes the issue of subjecting the material and electronic components to thermal stress across the entire sheet.

Rather than adding existing electronic components to the circuit, several researchers have investigated how to fabricate electronic components from scratch. Lewis et al. [11], for instance, pioneered 3D printing an LED. A 3D printer for electromagnetic devices [17] showed how motors and solenoids can be created by winding coils around 3D geometry during the printing process. However, since 
most electronic components cannot yet be created with personal fabrication tools, the fabricated objects still need to have commercially available electronic components, such as a microcontroller, added to their circuit after fabrication.

\subsection{Augmenting Existing Fabrication Machines}

Several research projects have augmented existing fabrication machines with additional hardware to perform new tasks. One of the first extensions of fabrication machines was to augment laser cutters with a camera to be able to detect the material on the fabrication bed (LaserCooking [3], PacCam [19]). More recently, researchers created hardware add-ons to fabricate new types of geometries, for instance, by adding a mill head to an existing 3D printer (ReForm [33]). A mill can also be used to undo previous fabrication steps, by removing formerly created geometry (Patching Physical Objects [26], Scotty [14]). Researchers have also modified the existing stationary build plate of $3 \mathrm{D}$ printers and replaced it with a rotating platform to allow 3D printing onto objects at an angle (Patching Physical Objects [26], Revomaker [4]). To assemble 3D printed parts into more complex mechanisms, researchers created an extension to a 3D printer's head that can pick up parts from the platform and place the parts into a new position (3D Printer Head as a Robotic Manipulator [7]). Finally, researchers added custom hardware add-ons to existing fabrication devices to investigate how to fabricate electronic components from scratch. A 3D Printer for Interactive Electromagnetic Devices [17], for instance, uses a wire delivery mechanism to wind coils thereby creating a first set of 3D printed electromagnetic devices.

Jubilee [30] highlights that building fabrication machines is a significant obstacle to creating novel fabrication processes facing researchers. While this motivates augmenting existing platforms rather than building them from scratch, this introduces the challenge of how to synchronize the add-on's functionality with that of the existing platform. Xprint [32] underlines that hardware constraints and non-open source firmware remain major challenges to integrating new fabrication processes into commercial platforms. Existing hardware add-ons, such as those mentioned in the previous section, have thus mostly relied on open-source hardware: A 3D Printer for Interactive Electromagnetic Devices [17], for instance, modified a 3D printer's software control files to accept their custom G-code for controlling their wire-coiling add-on. Similarly, Patching Physical Objects [26] used an open-source implementation of the MakerBot 3D Printer firmware to control the timing of the added mill, pump, camera and 5-axis rotating platform. However, relying solely on open-source implementations significantly limits the number of fabrication devices that can be augmented.

To be able to augment existing fabrication devices without the need to modify the underlying firmware, we developed a new motion-based signaling technique. Our technique embeds signals into the fabrication file that result in specific movements of the fabrication head, which can then be sensed with a motion sensor (accelerometer) attached to the fabrication head. Such signals can inform the hardware add-on when to start and stop its operation since they represent which part of the fabrication file is currently executed.

\section{LASERFACTORY}

LaserFactory is an integrated fabrication process based on a commercially available laser cutter that has been augmented with a silver dispenser and pick-and-place mechanism to support the manufacture of fully functioning electronic devices without human intervention. Figure 2 illustrates the LaserFactory fabrication process: After users download the design file, load it into the standard laser cutter software, and initiate the laser cutter job, LaserFactory first cuts out the device geometry using the regular functionality of the laser cutter (Figure 2a). Next, LaserFactory's silver dispenser deposits silver for the circuit traces (Figure 2b). Following this, LaserFactory's pick-and-place head picks up components from the component storage inside the laser cutter and assembles them onto the circuit traces (Figure 2c). Finally, the laser cures the traces which rigidly connects the components to the circuit (Figure 2d). Once LaserFactory completes these steps, the device is fully functional; here, the quadcopter readily flies off the fabrication platform (Figure $2 \mathrm{e}$ ).

In the following sections we describe each of the four main contributions in order. We start by describing the hardware add-on that incorporates a silver dispenser and a pick-and-place mechanism. Next we explain the laser soldering technique. After this, we present our motion-based signaling technique and finally we illustrate an end-to-end design and fabrication workflow based on our design and visualization tool coupled with the translation pipeline that converts the design into machine instructions.

\section{HARDWARE ADD-ON: SILVER DISPENSER AND PICK-AND PLACE MECHANISM}

We developed an add-on for a laser cutter that allows it to fabricate functional electromechanical devices. Our hardware add-on complements the laser cutter's native abilities to create the geometry of a device with circuit creation and assembly capabilities. We accomplish this by adding a silver dispenser and a pick-and-place mechanism to the laser cutter head (Figure 3).

\subsection{Add-on Functionality: Circuit Trace Creation and Component Assembly}

Our hardware add-on together with the laser cutter's native functionality creates functional devices in the following way: (1) To create the geometry, our system uses the regular laser cutter head to cut 2D geometries and optionally bend them into 3D using techniques, such as LaserOrigami [15]. (2) To add circuit traces, our silver dispenser in the form of a syringe extrudes silver paste. It can create highly conductive circuit traces down to $(3.2 \Omega / \mathrm{m})$ sufficient for most electronic components. (3) To assemble components, a pickand-place mechanism consisting of a second syringe on the add-on enables moving components inside the laser cutter. Components are picked from a storage area within the laser cutter platform. Our add-on accommodates lifting components up to a mass of $65 \mathrm{~g}$ and small SMD components down to size 2010. (4) Finally, we use laser soldering (explained in section 6) to cure the traces and make them conductive using the existing laser. The housing of the add-on was 3D-printed using an Ultimaker 3. With all onboard parts, the add-on weights $550 \mathrm{~g}$ and costs $\$ 150$ in parts. 

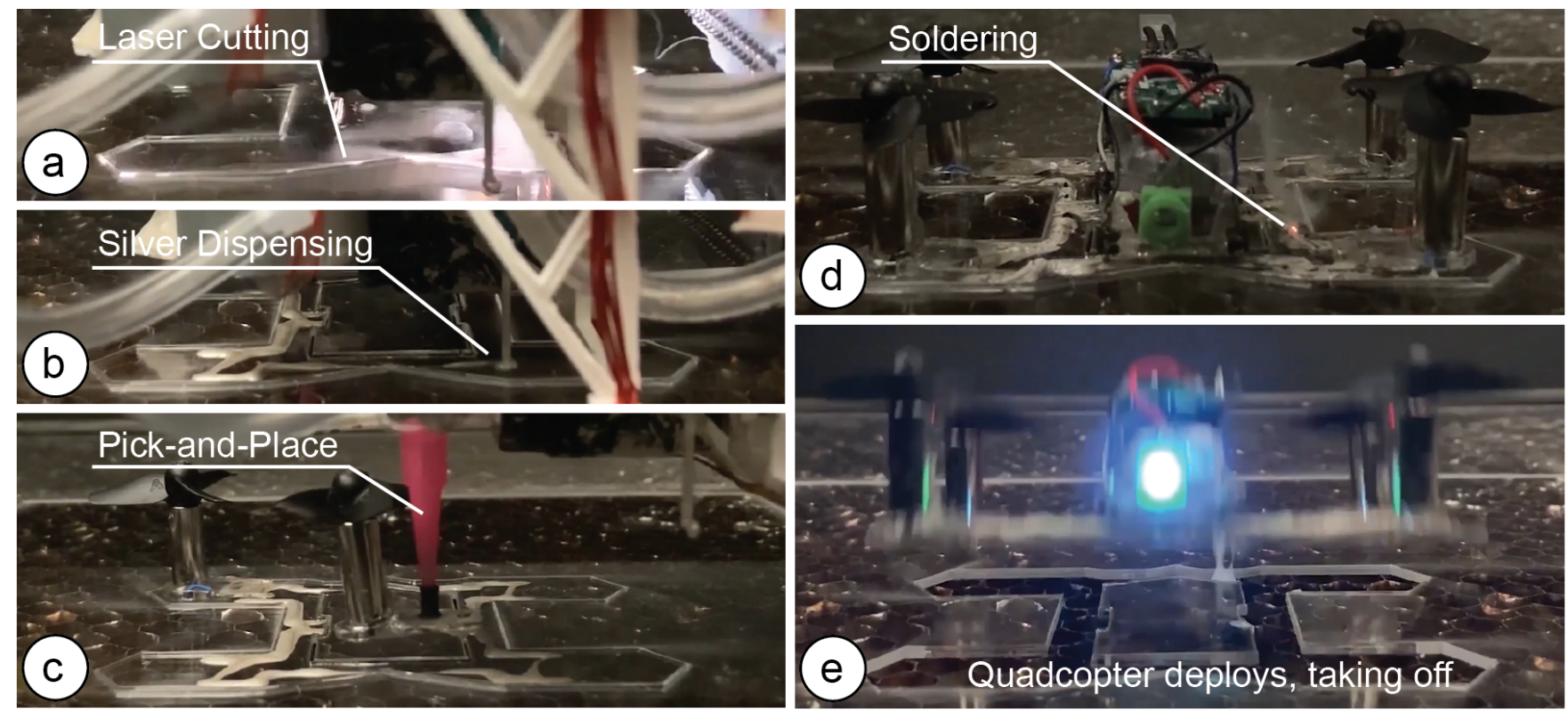

Figure 2: Making a Device with LaserFactory: (a) Cutting the device geometry, (b) dispensing silver to form the circuit traces, (c) picking-and-placing the components, here a quadcopter's rotor, and (d) curing the uncured silver traces. (e) When the last trace is cured, the device is fully functional; here, the quadcopter lifts directly off from the platform.

\subsection{Hardware Implementation}

Pneumatic Actuation for Dispensing Silver and Picking/Placing Components: The add-on's silver extrusion and pick-and-place mechanisms are both actuated pneumatically. The silver extruder consists of a $3 \mathrm{ml}$ syringe tipped with a 18-gauge needle filled with silver paste. The pick-and-place includes a second $3 \mathrm{ml}$ syringe connected to a rubber-tipped 14-gauge needle. For both dispensing and pick-and-place, we use a single pneumatic pump (TCS Electrical, $6 \mathrm{~V}$-rated) that supports both mechanisms by regulating air supply via a network of three identical 3-port, 2-position solenoid valves (Phoncoo, 6V-rated).

Vertical Actuation for Picking-and-Placing Components from the Fabrication Bed: The pick-and-place syringe must travel vertically during its operation, descending to the fabrication bed to pick/place components, and ascending during traveling to avoid collisions with other components. The pick-and-place syringe is actuated vertically by a NEMA 14 stepper motor (StepperOnline) via a lead screw and rigid coupling, to which it is connected in serial via a springloaded linear potentiometer (Sensata 9600). This affords the pickand-place mechanism the compliance required to avoid damage while allowing the controller software to evaluate the contact forces during component handling. It is also rigidly attached to a second linear potentiometer (BOURNS) which allows sensing of its height and thus distance to the material sheet in real-time.

Control Logic of Add-on: To enable the control logic for the addon, we mounted a micro-controller (Arduino Mega 2560) onto the add-on and shielded it with a custom PCB that houses the supporting electronics required for the add-on. The supporting electronics include one motor driver (Adafruit DRV8833), one 6V buck regulator (Pololu D24V25F6), and the accelerometer (part of a Sparkfun
LSM9DS1 IMU). To switch the pump between the silver dispensing syringe and the pick-and-place syringe, three parallel NPN transistors (2N2222) are used for overcurrent protection, each fitted with $1.5 \Omega$ series ballast resistors to avoid thermal runaway.

\subsection{Technical Evaluation of Hardware Add-on}

We evaluated both the silver deposition and pick-and-place capabilities to provide insight into which type of circuits can be created with LaserFactory.

Silver deposition trace characteristics: Since the needle gauge has a significant impact on the minimum trace width, we ran an experiment to determine the smallest needle gauge through which our $6 \mathrm{~V}$-rated pump could dispense silver. Smaller needle gauges require higher pressures to dispense the highly viscous silver. We found that by testing needle gauges from 14 to 21 , that 18 was the highest gauge through which our 6V-rated pump was able to dispense silver. We then tested depositing this silver two ways. First, by depositing directly onto a material substrate, which facilitates rapid device fabrication but allows silver to spread across the substrate over time, increasing trace widths up to $3 \mathrm{~mm}$. As a second slower alternative, we engraved $0.75 \mathrm{~mm}$ channels using a defocused laser and dispensed silver into these, yielding a minimum trace width of $0.75 \mathrm{~mm}$ with our current setup. For the future, this trace width could be further reduced by using a stronger pump and narrower nozzle; Valentine et al. [29] for instance, use a $0.2 \mathrm{~mm}$ nozzle with their platform.

After determining the minimum trace width, we also determined the minimum distance between two adjacent traces, which is important since it determines the pin spacing and thus the size of the components that can be used. To find the minimum trace distance 


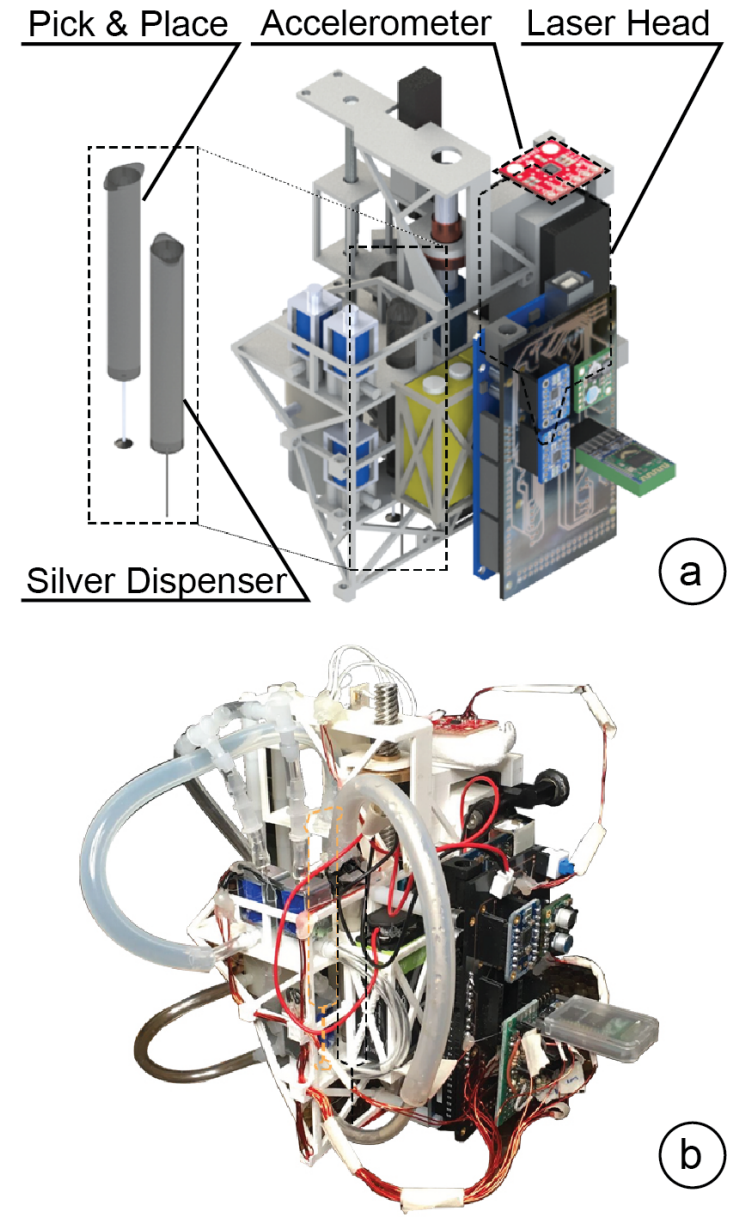

Figure 3: Our hardware add-on shown as (a) a CAD rendering and (b) a photo of the physical device. The add-on consists of a silver dispenser to create circuit traces and a pickand-place mechanism to assemble electronic components. It attaches to the laser head, which is used to create the device's geometry and to cure the circuit traces. The image also shows the accelerometer used for detecting the motion signaling when the add-on should start/stop its operation.

between adjacent traces, we deposited and soldered parallel traces spaced between $0.4 \mathrm{~mm}$ and $1.8 \mathrm{~mm}$ apart in $0.2 \mathrm{~mm}$ increments. We found that the minimum distance was $0.8 \mathrm{~mm}$ between traces for silver that had been appropriately refrigerated before use, as the silver's high viscosity and surface tension prevents it from spreading after it has been dispensed. However, for silver left at room temperature, $1.5 \mathrm{~mm}$ was the minimum distance achievable with our chosen needle gauge. Smaller distances may cause short-circuits between adjacent traces due to the silver spreading across the substrate or ballooning slightly during laser curing. In addition, while our silver dispenser negatively pressurizes to de-ooze before moving between traces, it is not actuated in the $\mathrm{z}$-axis and can therefore not retract from the substrate, leading to silver occasionally being spread between traces. This, however, does not cause short circuits or impact the device functionally since silver only becomes conductive where addressed by the laser which only targets the actual traces, thus the remaining silver can be wiped off. For future work, we plan to make the dispenser retractable to avoid additional spread. Based on these results, we conclude that we can currently create traces fine enough to work with SMD components down to a $1.55 \mathrm{~mm}$ pin spacing in ideal conditions where the silver was refrigerated before use, and $2.3 \mathrm{~mm}$ when silver is at room temperature, which can be used for components with standard pin spacings of $2.54 \mathrm{~mm}$.

Pick and place component criteria: We evaluated the criteria under which LaserFactory can pick and place components. First, components require a minimum flat surface area of $3 \times 3 \mathrm{~mm}$ above the component's center of mass for the suction tip's $2.5 \mathrm{~mm}$ diameter nozzle to form a good contact. We found this could be increased or decreased by increasing or decreasing the diameter of the pick-andplace nozzle, respectively. Second, components must have a mass smaller than $65 \mathrm{~g}$ for the pump suction to lift them. This threshold allows lifting large components, such as an Arduino Mega microcontroller $(37 \mathrm{~g})$. A stronger pump could further raise this threshold. Third, their height cannot exceed $27 \mathrm{~mm}$, currently limited by the maximum extension of the linear potentiometer used to assess picking height. Given these constraints, LaserFactory can pick large, heavy components, such as typical $3 \mathrm{~V}$ batteries; tall components such as rotors; and a variety of small components, such as SMD resistors down to size 2010. For future work, these constraints can be further lifted by using a smaller nozzle, larger pump, and longer lead screw.

Use over time: During the course of fabrication, the peak power draw measured from the two on-board parallel 9V lithium ion batteries was $13.5 \mathrm{~W}$. Between no-load cases of cutting/folding and variable-load cases of PCB-making, this is sufficient for approximately 4 hours of continuous device creation. We detected no irregularities in laser carriage motion that would indicate undue stress on the laser cutter during use.

\section{LASER SOLDERING: CURING CIRCUIT TRACES USING A CO2 LASER}

When both the silver paste has been deposited and the components have been placed onto a substrate in the laser cutter, the silver is not yet conductive, nor does it rigidly bond components to the substrate. For this, the silver requires curing via heating, traditionally achieved via baking the created device in an oven before it becomes functional. However, this has several drawbacks, such as that the heat in the oven can damage the material substrate and the electronic components due to the thermal stresses. To solve this issue, we developed a technique that uses the laser to cure the circuit traces and connect components electrically. A benefit of using the laser is that it allows for a fully integrated fabrication process with no manual work required. In addition, heat is only applied locally to traces and thus affects the material sheet only in the locations of the trace. Finally, using the laser is fast, approximately 5 minutes in contrast to the 2 hours achieved via uniform heating in an oven [29]. Once the last circuit trace is cured, the device is functional right off the fabrication bed. 


\subsection{Fabrication Settings for Laser Soldering}

We ran a set of experiments to determine the best power, speed, and z-axis settings for curing silver traces. For the experiments, we used a Universal Laser Systems ULS.PLS.150D laser cutter equipped with a 2.0 inch lens. For the silver, we used the silver paste from SunChemical (C2180423D2), which has a composition of $56.69 \%$ $24 \mathrm{~K}$ silver and $43.31 \%$ a blend of resin and solvents.

Power/Speed/Height Settings: To find admissible laser cutter settings that successfully cured the silver, we dispensed a series of $100 \mathrm{~mm}$ silver traces, then soldered them with different parameters. We first calibrated the vertical offset that produced a defocused laser spot diameter equal to trace width of silver. We then irradiated the silver using permutations of power and speed settings on the interval $0.2 \%$ to $90 \%$, recording the resistance of the traces using a Greenlee DM-820A Multimeter and recording whether it was viscous (uncured), solid (cured), or burnt (overcured) to touch. We found numerous admissible power-speed combinations producing rigid, conductive traces. These are bounded by the combination of $0.2 \%$ speed with $7 \%$ power on the lower bound, and the combination of $7 \%$ speed with $90 \%$ power on the upper bound. Linear interpolation between these lower and upper bounds yield further admissible settings, showing that lower powers require commensurately lower speeds to effectively cure the silver. Power/speed ratios greater than this produce charred, brittle traces, while power/speed ratios lower than this leave the silver poorly conductive and uncured. In addition, high speeds above $7 \%$ produce traces that remain subcutaneously uncured regardless of power, while powers below $1 \%$ do not elevate temperatures sufficiently to cure traces at all.

\subsection{Technical Evaluation of Laser Soldering}

To determine the conductivity of traces cured via laser soldering and the soldering connection quality between traces and components, we ran the following technical evaluation.

Conductivity: To evaluate the conductivity that laser soldering achieves in a single pass of the laser, we fabricated 8 traces of length $0.5 \mathrm{~m}$ and with a minimum trace width of $0.8 \mathrm{~mm}$. we then measured the resistance between the traces' ends using a Multimeter (Greenlee DM-820A). The resistance was normally distributed about $3.2 \Omega / \mathrm{m}$ with a standard deviation of 0.0005 . LaserFactory thus supports a range of electronic components, actuators, I2C communication, PWM, and medium to high-power applications.

Power and frequency: We used a current-limited power supply to pass DC current through five $100 \mathrm{~mm}$ traces at $1 \mathrm{~A}, 2 \mathrm{~A}$ and $3 \mathrm{~A}$ power without observing arcing or warming. In addition, we used a frequency generator to deliver an AC signal from $0 \mathrm{~Hz}$ to $100 \mathrm{kHz}$ in logarithmic increments. Investigating the signal with an oscilloscope showed there was no significant signal attenuation.

Soldering connection quality of traces and components: To test how strong the cured connections between the traces and the components are, we evaluated their mechanical rigidity. We used a two-pad component and hung weights to the face of the component to apply normal forces between $25 \mathrm{~g}$ and $300 \mathrm{~g}$ in increments of $25 \mathrm{~g}$. We found that a normal force of $225 \mathrm{~g}$ was required to disconnect the component from the trace. The strength of the curing connection is considerably less than that of a traditional solder connection. While this can be a drawback for devices that undergo high mechanical strain, it also has the benefit that components can be re-used, as users can pull them off with reasonable force.

Warping: Warping is a form of material distortion that can occur during laser cutting, and can arise from laser soldering too. This occurs due to rapid local temperature changes from selective heating of areas being targeted. The amount of warping is affected by both the choice of material and the part size, as heat cannot dissipate out of smaller parts via conduction. To explore this, we tested its effect on three materials: extruded acrylic, and two cast acrylics (unbranded plexiglass). All were of equal transparency and thickness $(1.5 \mathrm{~mm})$. While we observed no warping for the cast acrylic, we found that extruded acrylic did exhibit warping, and we calibrated our laser cutting settings to minimize its effects. Since some of our devices are made from extruded acrylic, this warping can be observed as a result of the laser soldering process in the accompanying video. While this distortion can impact both the visual appeal of devices as well as their operation if their function relies on precise planar surfaces, the effects can be largely avoided by using one of the alternative materials. In addition, we found that warping can be further reduced by cutting the device out in the final stage in the fabrication process instead of at the beginning, allowing the surrounding material to hold it in place during soldering. Alternatively, connector tabs can be left on the cut outline to achieve the same effect, i.e. keeping the device attached to the sheet during soldering, and cutting these tabs to release the device at the end.

Materials: We tested LaserFactory's compatibility with a range of materials, including extruded acrylic, cast acrylic, leather, cardboard, hardwood (oak) and plywood. Extruded and cast acrylics were compatible with the full LaserFactory suite, including the engraving of trace channels (4.3) and bending of the substrate (5.3) to create $2.5 \mathrm{D}$ geometries; however, extruded acrylic was prone to warping. The woods and and cardboard were also tested successfully, although these char visibly during soldering if the laser contacts the material directly. Furthermore, while not susceptible to warping, they cannot support bending or the engraving of trace channels. Lastly, leather and other soft textiles were not able to support the silver traces due to the traces cracking upon being bent after curing. This, however, can be addressed by attaching a firm backing to the soft material.

In summary, we conclude that laser soldering using a $\mathrm{CO} 2$ laser is suitable for creating highly conductive traces, that the soldering connection holds components in place while allowing components to be removed using manual force, and that warping due to laserinduced local heating only affects certain materials significantly.

\subsection{Creating Non-planar Geometries}

In this section, we report on two proof-of-concepts that showcase different methods of creating non-planar geometries using LaserFactory.

Laser Soldering Folded 2.5D Circuit Traces: Since our technique is built based on a laser cutter, which has been shown to not only be able to create $2 \mathrm{D}$ geometries but also $3 \mathrm{D}$ geometries via folding (LaserOrigami [15]), we also investigated if we can dispense and cure circuit traces that are positioned across a fold, which would allow LaserFactory to fabricate 2.5D geometries with embedded 
traces and electronics (Figure 4a). Our early experiments showed that by using the laser to slowly heat traces across a hinge, the silver remains viscous and uncured, which allows the substrate to become compliant and fold under its own gravity. By continuing the heating process after folding, the silver gradually cures and hardens, producing $2.5 \mathrm{D}$ geometries with embedded traces. While we did not evaluate the different power/speed settings in a controlled experiment, we did not observe a difference in resistance between folded and planar traces. We include the associated laser settings in Table 1.

Cutting and stacking layerwise 3D structures: In addition to folding $2.5 \mathrm{D}$ traces, we report on a second method of creating $3 \mathrm{D}$ geometries that leverages the unique combination of our pick-andplace mechanism with a laser cutter. In addition to placing electrical components on a device, we can also pick-and-place pieces of the material substrate. To harvest these pieces, shapes are first cut from the substrate and then the pick-and-place is used to stack them to create 3D structures (Figure $4 \mathrm{~b}$ ). By discretizing a structure into layers, these layers can be cut using a focused laser, stacked via pick-and-place, and welded using a defocused laser [27] to produce rigid 3D structures without manual intervention.
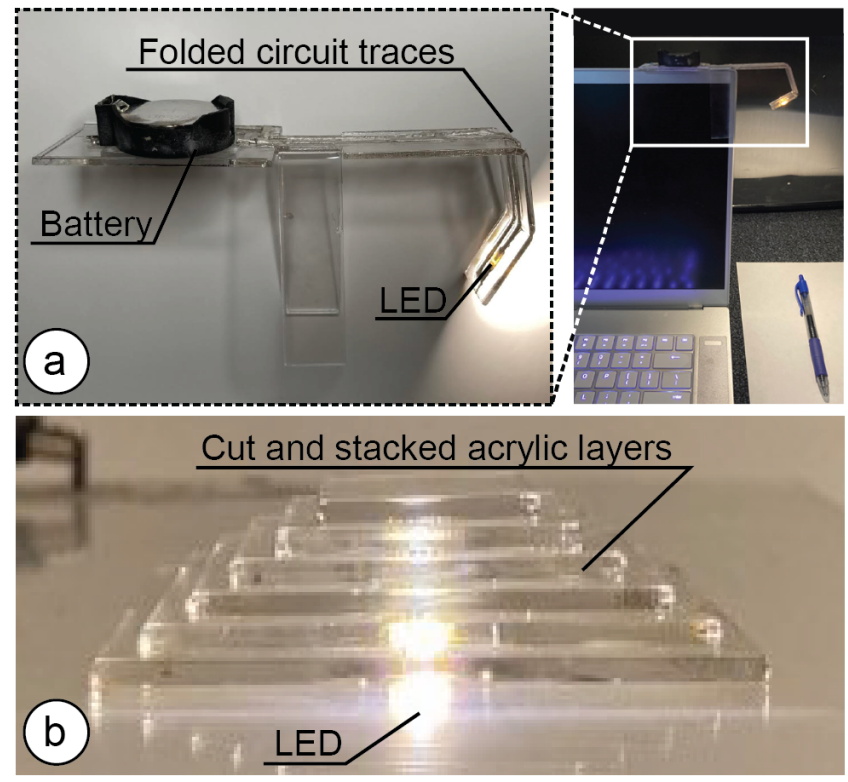

Figure 4: LaserFactory can be used to create (a) 2.5D geometries via folding, (b) as well as discretized 3D geometries via cutting and pick-and-placing the material substrate itself.

\section{AUGMENTING A LASER CUTTER THROUGH MOTION-BASED SIGNALING}

Augmenting existing fabrication devices reduces engineering effort by piggy-backing existing platforms rather than building hardware/software from scratch, and by making the new functionality available to a wide range of users who already own or operate the platform. However, interfacing with a commercial fabrication platform that is not built to accommodate an expansion of its functionality is challenging [30,32]. In building our hardware augmentation to a laser head, we investigated solutions that do not require access to the proprietary software of the fabrication platform and that therefore could potentially be platform-agnostic to permit augmenting a range of different fabrication devices. To this end, our method involves two primary stages. First, our method embeds additional lines in the fabrication file. These include additional paths such as trajectories for navigating between electrical components, and also specific motion patterns that signal instructions to the add-on. Second, our method detects these motion patterns using a motionbased classifer based on accelerometer data running locally on the add-on. Our method to transmit fabrication instructions thereby relies only on the fabrication platform's ability to execute motion in the X-Y plane; the add-on has no explicit knowledge of the contents of the fabrication file itself. In the next section, we provide more details on how we create the additional lines to produce carriage motion of the laser cutter and how we signal the transition between different fabrication stages using the motion patterns in the design file.

\subsection{Creating Carriage Motion by Embedding Additional Lines in the Design File}

To generate carriage motion without accessing the laser cutter's firmware, we add extra lines into the design file that is sent to the laser cutter. Figure 5 illustrates this using a quadcopter design file that has additional lines embedded for dispensing silver, pick and place, and soldering. The lines are offset in the design file to compensate for the add-on's position with respect to the laser head. The original design file as created by a user can be seen in Figure $8 \mathrm{~g}$.

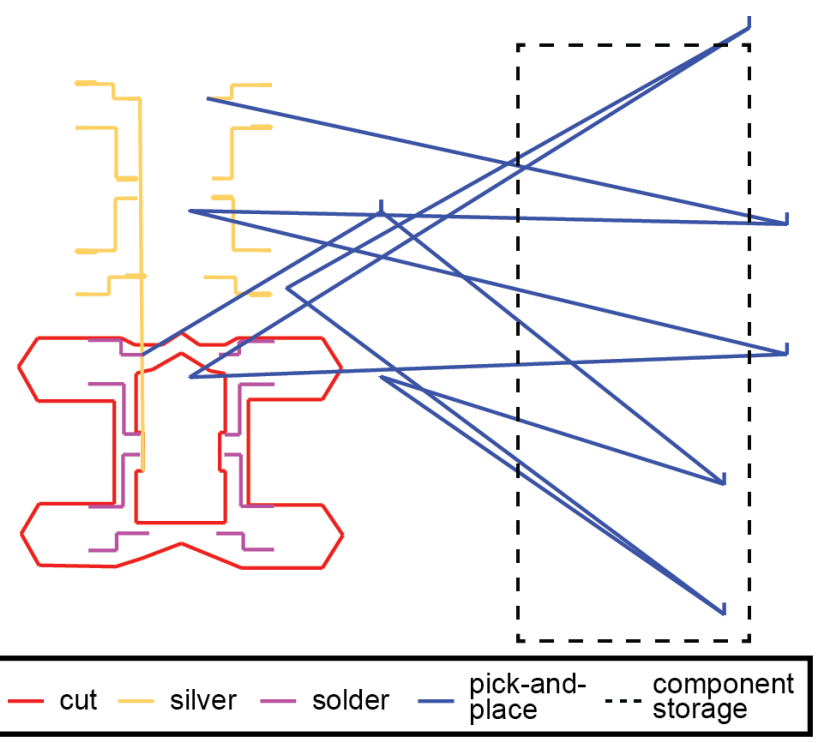

Figure 5: Adding lines to the design file to create additional carriage motion for pick-and-place operations, silver dispensing, and soldering. Lines are offset to compensate for each add-on part's position with respect to the laser head. 


\subsection{Signaling Fabrication Instructions using Motion Patterns in the Design File}

After additional lines have been embedded to create trajectories that guide the laser head to execute silver dispensing and pick-andplace manoeuvres, a separate step involves relaying fabrication instructions to the add-on (when to start and stop silver dispensing and pick-and-place maneuvers) without communication with the laser cutter. This involves first embedding additional lines that encode these fabrication instructions, and then interpreting these using sensors. In choosing sensors, we disqualified candidates that could not be entirely integrated into the add-on, such as external position tracking. We also disqualified candidates that may introduce platform-specific dependencies, such as onboard cameras or position sensors that measure the inside volume of the fabrication platform.

On the other hand, an accelerometer affixed to the laser head measures only its inertial motion in terms of acceleration, which makes it possible to recreate on other platforms. By appending a unique line pattern to the beginning and end of paths in the fabrication file, we elicit a specific motion from the laser head, which the onboard accelerometer interprets in order to start or stop a fabrication procedure (silver deposition or pick-and-place).

Motion Pattern Shape: To select a motion pattern for our signal, we investigated the motion of different shapes. In particular, we considered the motion of straight lines, squares and circles in order to test independent, sequential, and simultaneous motions in the $\mathrm{X}-\mathrm{Y}$ plane, respectively. While all candidates yielded a uniquely classifiable signal, we found that squares and circles were unsuitable as performing their motions may cause extrusion of silver outside of a trace or collision with other components. A one-dimensional line that programmatically tracks backward along a previously deposited trace, in contrast, provides a trajectory that keeps the tip and any dispensed silver above traces.

Duration of Line Pattern: The motion pattern's duration, and therefore physical length, must be small enough to be quickly evaluated by the classifier to allow rapid transmission of fabrication instructions yet long enough for the digital sampling to produce sufficiently many data points for the classifier. We tested the execution of straight lines of lengths between $0.5 \mathrm{~mm}-6 \mathrm{~mm}$ in $0.5 \mathrm{~mm}$ increments, measuring the duration of each. For our processor's maximum sampling speed of $300 \mathrm{~Hz}, 3 \mathrm{~mm}$ lines constituted the fastest signal (150ms) that had a sufficient number of data points ( 45 samples) to encode a uniquely identifiable signal. By accelerating along the trace for $3 \mathrm{~mm}$, then decelerating to stop, the signal elicits two consecutive equal but opposite spikes on the accelerometer. This creates a unique signal, as no other maneuver requires retracing the exact same path, that can be reliably classified using the accelerometer (Figure 6).

\subsection{One-time signal calibration and Real-Time Classification}

To build the classifier, we perform a one-time calibration of the $3 \mathrm{~mm}$ line signal described above. We perform this calibration by executing the pattern and measuring the corresponding linear accelerations from the accelerometer in the X-Y plane. We sample the

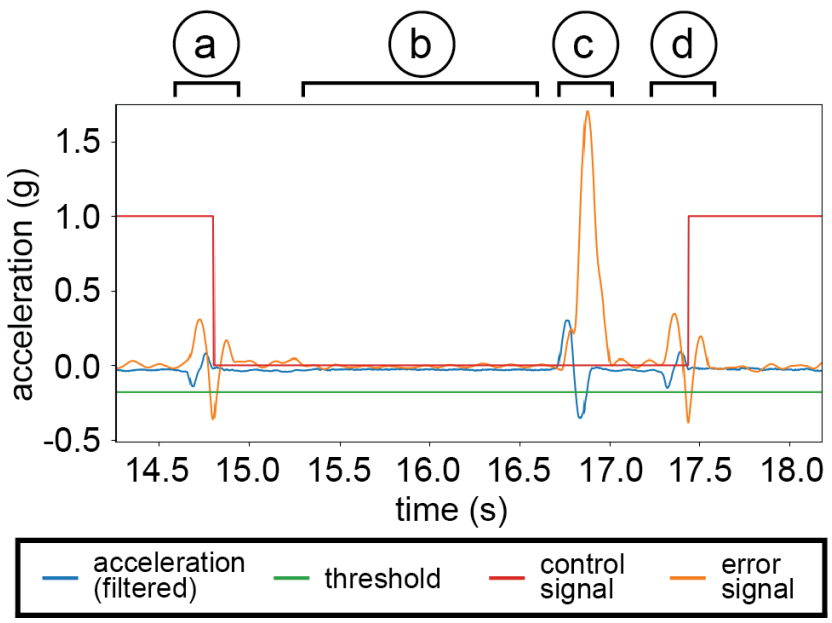

Figure 6: Filtered accelerometer data during execution of the embedded pattern. When the pattern is executed, the (blue) acceleration produces oppositely signed spikes in rapid succession. (a,d) This characterized template causes the (orange) error signal to drop below the (green) threshold which flips the control signal to start/stop a command; here, starting/stopping the silver extrusion. (b) Periods of constant velocity are marked by accelerations around 0 ; (c) volatile maneuvers such as moving between contiguous traces causes large errors and thus can be differentiated from actual motion-signal instructions embedded in the fabrication file.

pattern at $300 \mathrm{~Hz}$ and low-pass filter it using a 24-point averaging filter to remove noise to construct the signal template.

To choose the error signal threshold to identify a signal, we built a dataset of true positives by sampling the signal pattern 100 times, recording the maximum negative error produced by the matched filter for each. Equivalently, we built a dataset of true negatives, compiling a further 100 measurements taken during manoeuvres not associated with a signal. Modeling each as a normal distribution $\mathcal{N}\left(\mu, \sigma^{2}\right)$, we found the mean and standard deviation for the true positives (X) and true negatives $(\mathrm{Z})$ were $X \sim \mathcal{N}(-0.311,0.002)$ and $Z \sim \mathcal{N}(0.057,0.008)$ respectively and choose our threshold at the intersection of these curves $(-0.18)$ in order to minimize misfirings (false positives and negatives).

After the one-time calibration, our add-on is ready for real-time detection of the motion signals embedded in the design file. For the real-time detection, we low-pass filter the raw acceleration data using a 24-point averaging filter and save it into a 45-point circular buffer. We then build a matched filter by evaluating this buffer against the signal template using the L2 norm at $300 \mathrm{~Hz}$. This produces an error signal of the low-pass filtered data that our detection algorithm normalizes around 0 . We then continuously evaluate the output of the matched filter and signal an instruction to the silver extruder or pick-and-place mechanism when the error signal surpasses a characterized threshold. 
Table 1: Fabrication settings. Columns, from left to right, show (a) process order (b) line manipulations, (c) laser cutter settings, (d) add-on settings for the silver dispenser and pick-and-place mechanism. A tilde indicates varying a value.

\begin{tabular}{|c|c|c|c|c|c|c|c|c|c|c|c|c|c|}
\hline \multicolumn{3}{|c|}{ Fabrication Process } & \multicolumn{2}{|c|}{ Line Manipulation } & \multicolumn{4}{|c|}{ Laser Cutter Settings } & \multicolumn{5}{|c|}{ LaserFactory Hardware Settings } \\
\hline Step & Primitive & Mandatory? & Offset $(\mathrm{X} / \mathrm{Y} \mathrm{mm})$ & Passes & Color & Power $(\%)$ & Speed $(\%)$ & Height $(\mathrm{mm})$ & Pump & Stepper & Valve 1 & Valve 2 & Valve 3 \\
\hline$\# 1 \mathrm{a}$ & Cut outline & Yes & $(0,0)$ & 1 & Red & 80 & 30 & 5 & OFF & OFF & OFF & OFF & OFF \\
\hline$\# 1 \mathrm{~b}$ & Raster footprints & No & $(0,0)$ & 1 & Black & 30 & 30 & 5 & OFF & OFF & OFF & OFF & OFF \\
\hline$\# 1 \mathrm{c}$ & Engrave channels & No & $(0.4,0.7)$ & 1 & Green & 70 & 25 & 22 & OFF & OFF & OFF & OFF & OFF \\
\hline$\# 2 \mathrm{a}$ & Dispense silver & Yes & $(-1.8,-68)$ & 1 & Yellow & 0 & 1.5 & 0.8 & ON & OFF & OFF & ON & ON \\
\hline$\# 2 \mathrm{~b}$ & Signal/De-ooze & Yes & $(-1.8,-68)$ & 1 & Yellow & 0 & 1.5 & 0.8 & ON & OFF & ON & ON & OFF \\
\hline \#3a & Pick & Yes & $(1.5,3)$ & 1 & Blue & 0 & 1.5 & $\sim$ & ON & $\sim$ & $\mathrm{OFF}$ & OFF & ON \\
\hline$\# 3 \mathrm{~b}$ & Carry component & Yes & $(1.5,3)$ & 1 & Blue & 0 & 1.5 & 28 & ON & OFF & OFF & OFF & ON \\
\hline$\# 3 \mathrm{c}$ & Place & Yes & $(1.5,3)$ & 1 & Blue & 0 & 1.5 & $\sim$ & ON & $\sim$ & ON & OFF & OFF \\
\hline$\# 4$ & Cure silver & Yes & $(1.5,3)$ & 1 & Magenta & 7 & 1 & 68 & OFF & OFF & OFF & OFF & OFF \\
\hline$\# 5 \mathrm{a}$ & Fold hinge & No & $(0,0)$ & 60 & Cyan & 30 & 100 & 68 & OFF & OFF & OFF & OFF & OFF \\
\hline$\# 5 \mathrm{~b}$ & Cut 2nd outline & No & $(0,0)$ & 1 & Orange & 30 & 100 & 5 & OFF & OFF & OFF & OFF & OFF \\
\hline
\end{tabular}

\subsection{Technical Evaluation of the Motion-Based Classifier}

We evaluate both how reliable our motion-based classifier can detect the motion-signals and if the motion-based classification approach transfers across different fabrication machines.

Performance: First, we evaluated the accuracy with which our motion-based classifier detects signals embedded in the fabrication file. To determine this, we mounted the add-on to the laser head, then started fabrication of a design consisting of 5 horizontal lines and 5 vertical lines, each $50 \mathrm{~mm}$ in length, totalling a combined 10 start signals and 10 stop signals (20 signals in total). For each signal, we noted whether detection was successful, and after all lines were executed, we dismounted the add-on. We repeated this procedure 10 times, for a total of 200 signals. One signal was undetected, yielding a classification accuracy of $99.5 \%$ for this test, with 0 false positives, and 1 false negative. We then executed fabrication of our three application examples; the quadcopter, wristband, and $\mathrm{PCB}$, five times each. These consist of a total of 465 signals, including 305 silver deposition sequences between $7 \mathrm{~mm}$ and $90 \mathrm{~mm}$ in length, and 115 pick-and-place sequences between 140 and 190mm in length. No signal misfirings were registered during these trials. By integrating the cumulative distribution functions of our normal distributions from Section 6 to evaluate the Receiver Operating Characteristic (ROC) curve for our characterized classification threshold, we find that our model predicts a false positive rate of approximately $0.2 \%$ and false negative rate of $0.1 \%$, which agrees with these results. This result shows that our procedure produces a reliable classification due to our ability to freely design a unique signal for detection. However, in case the detection misfires, LaserFactory currently has no way to detect it. We plan to explore such detection as part of future work by embedding additional lines that function as interrupts.

Transferability: We designed the hardware add-on to be fully self-contained and the fabrication signaling to be motion-based in order to demonstrate an add-on design that can be used to augment different fabrication machines. To test if our add-on can indeed work on different fabrication machines, we tested our approach on the 3D printer Ultimaker 3 . To conduct this test, we wrote a custom G-code script that mimicks silver deposition by executing first a vertical then a horizontal $30 \mathrm{~mm}$ line, with our $3 \mathrm{~mm}$ signal pattern appended at every line end. We use the G0 command to execute the pattern, representing a co-ordinated movement at rapid rate without material extrusion with feed rate set to F15000. We deployed this file 10 times on the Ultimaker 3 and achieved a $100 \%$ signal classification accuracy. While additional tests on other fabrication devices are needed before full transferability can be claimed, the results show that motion-based signaling has the potential to work across different fabrication devices. Before motion-based signaling can be used on a new machine, the motion-signal must first be characterized via the one-time calibration and the add-on's physical clips need to be adjusted to the differently shaped tool head.

\section{DESIGNING A DEVICE FOR USE WITH LASERFACTORY}

To support the creation of devices with LaserFactory, we provide the end-to-end fabrication pipeline shown in Figure 7. It consists of a design and visualization tool as well as a post-processing script that converts the design file into a set of machine instructions for fabricating the device with LaserFactory on the augmented laser cutter. The design tool lets users create 2D designs using drawing tools for the geometry ('cut', 'fold'), the circuit traces ('wire'), and for placing electronic components ('place part'). This allows users to concurrently design the geometry and the electronic circuit. The accompanying visualization tool renders both the final $2 \mathrm{D}$ or $3 \mathrm{D}$ design and allows users to animate each step of the fabrication process. This assists users who may find it difficult to visualize the abstract $2 \mathrm{D}$ drawing. In addition, it allows users to inspect the order of each step in the fabrication process. On export, our post-processing script automatically translates the design file into machine instructions. Users do not have to add any explicit instructions for the add-on.

\subsection{Design Tool}

Our design tool (developed as a plugin to the 2D editor Adobe Illustrator) supports users in placing components, drawing circuit traces and designing the geometry of their devices. Figure 8a shows the toolbar of our design tool, which has the following functionality.

Electronic component library: To facilitate circuit design, users can select electronic components from a library of parts. Common components can be accessed directly from the toolbar and others can be found using the 'find part' button that opens the component library (Figure 8a). After selecting a component, it is loaded as a 2D representation onto the canvas (Figure $8 \mathrm{~b}$ ). Components appear as their physical footprint superimposed with their electronic symbol. Seeing the electronic components allows users to appraise the size 


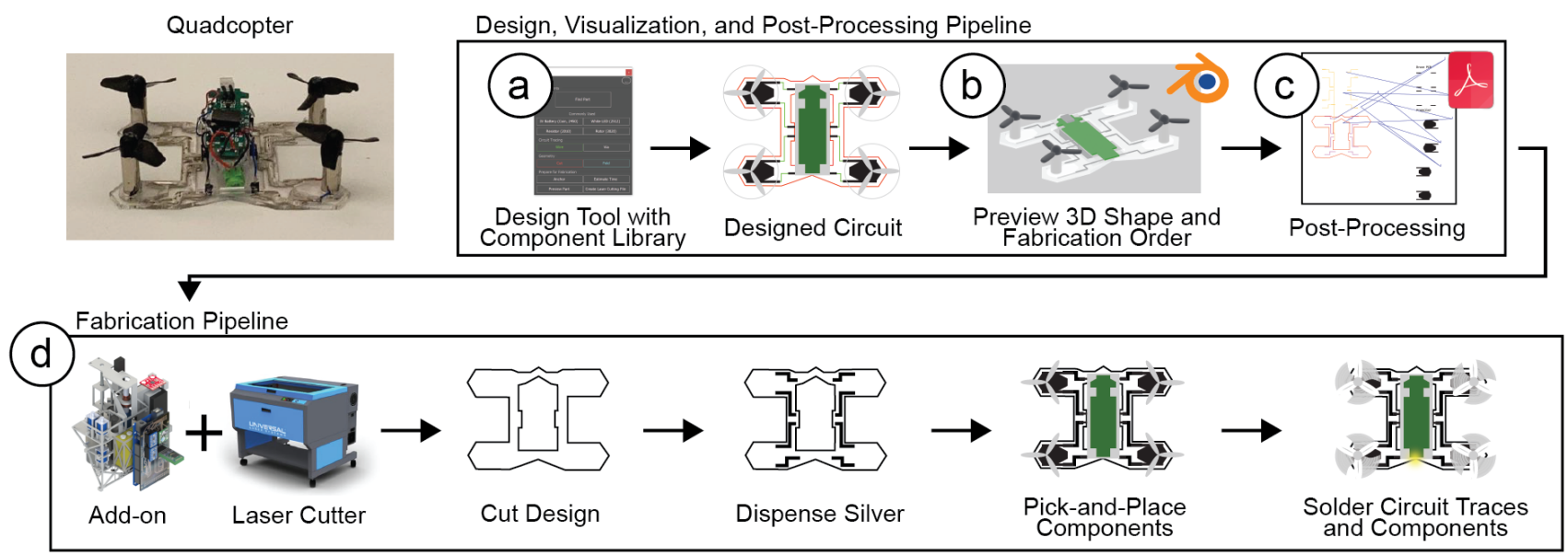

Figure 7: LaserFactory Pipeline: (a) Design Tool: Users place components, and draw geometry and circuit traces. (b) Visualization Tool: Users can preview the design in 3D and visualize the fabrication steps for debugging the design. (c) Post-Processing: On export, the design file is converted into machine instructions for the augmented laser cutter. (d) Fabrication: The augmented laser cutter then executes the post-processed file, i.e. cuts the geometry, dispenses silver for circuit traces, pick-and-places components, and then cures the silver to make the traces conductive and solders the components. Once fabricated, the device is fully functional.

of the main geometry in their design and adjust the design as needed.

Wire drawing tool: LaserFactory offers a wire drawing tool (Figure $8 \mathrm{~b}$ ), which is used to connect electronic components with traces. While free-form traces are possible, we enforce the wire tool to snap to $0 / 90^{\circ}$ in order to align signaling motion with trace geometry for cleaner fabrication. Traces can also snap to the electrodes of components on approach to them, ensuring good electrical connections during fabrication. In the drawing, circuit traces are differentiated from other types of lines by color coding them in green (Figure 8g).

Geometry drawing tools: The LaserFactory user interface offers two drawing tools to define the geometry of the object (Figure 8c): the cut drawing tool and the bend drawing tool. In the drawing, cut lines are visualized in red and bend lines are visualized in cyan. For bending, users only have to indicate where to bend, but not in which order, which is taken care of by our post-processing script. For bending, users must use the 'anchor' tool to assign a section as the anchor plane which constrains this section as the one that remains planar in the event of folding.

Export tools: Finally, the interface includes a set of tools for use on completion of the design (Figure 8d). The "estimate time" tool parses the file to estimate the total fabrication time that is then displayed in the view. The 'Preview Part' tool opens the visualization tool, which we describe in more detail in the next section. Finally, the 'create laser cutting file' tool post-processes the design for fabrication and generates the final fabrication file.

\subsection{Visualization Tool}

Since the 2D drawing is an abstract representation of the resulting design, we developed a 3D visualization tool (developed as a plugin to the $3 \mathrm{D}$ editor Blender) that users can launch at any time in the design process. Figure 9 shows the visualization tool, which has the following functionality.

Rendering the $3 D$ design: The visualization tool shows the design as a solid 3D geometry, including any folded parts. This allows the user to see if the folds are placed correctly and result in the desired 3D geometry. In addition, all electronic component footprints are replaced with their respective 3D models, providing a preview of the final device.

Order of fabrication steps: Besides showing the design as a 3D shape, the visualization tool also contains a video playback that animates each step of the fabrication process. This allow users to double check if the post-processed design file contains the correct fabrication order. For instance, folded geometry must be released with a cut first before any folding can occur, which can be observed in the animation.

Visualizing the movement of the tool head: Finally, the visualization tool also renders the tool used in each fabrication step: the laser during cutting and curing (and any folding), the silver dispenser during trace creation, and the pick-and-place nozzle during component placement. This allows users to assess potential collision in their design, such as unwanted intersections between the laser beam and placed components to prevent damage.

\subsection{Post-processing of Design File}

When exporting the design file, a number of post-processing steps are applied to the design file to make it work with the LaserFactory add-on. The user's exported design contains only the cut and bend lines, the circuit traces, and the locations of components on the device. In a first post-processing step, additional motion lines are added for picking-and-placing components and curing the traces. 


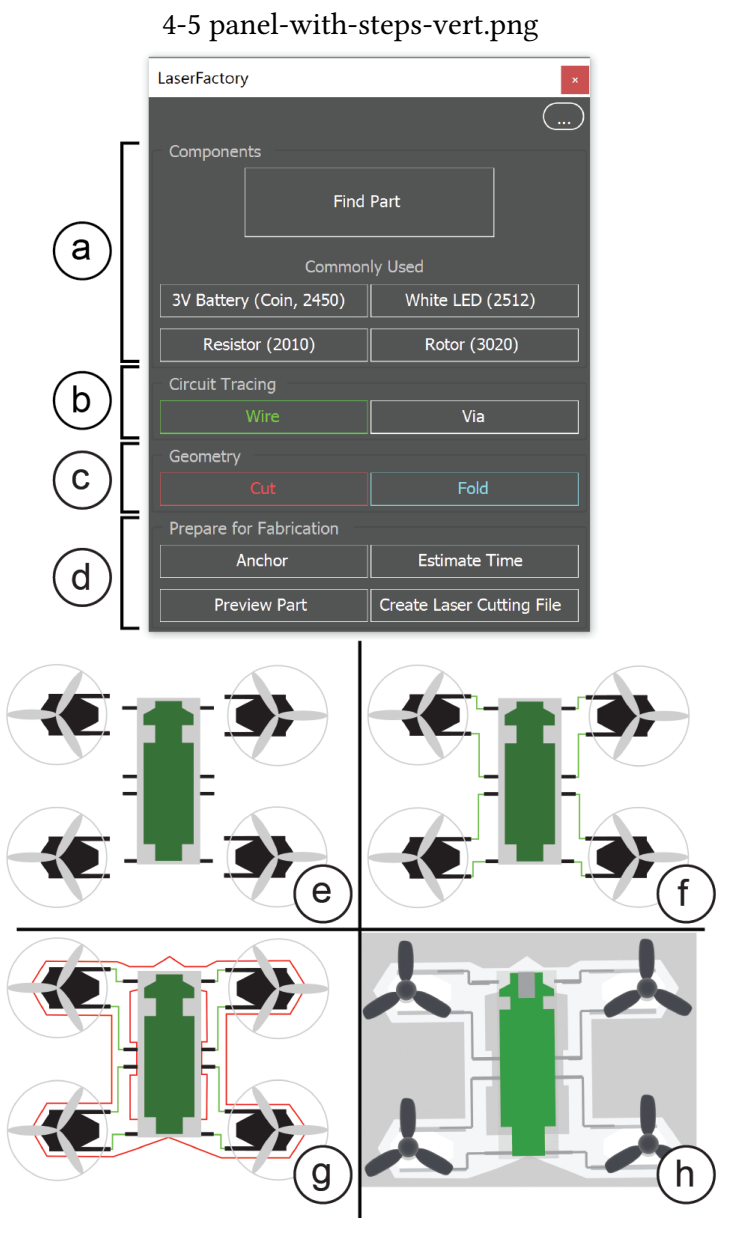

Figure 8: Users use the design toolbar to design a device such as a quadcopter. The steps involved $(a, e)$ placing components from a part library, $(b, f)$ routing circuit traces, $(c, g)$ drawing lines for the geometry, here showing cut lines and $(d, h)$ using export tools, here showing rendering the output of the visualization tool.

Next, all lines that belong to the silver dispenser and pick-andplace head are offset by each add-on's distance to the laser head. In addition, all curing lines are offset by a small amount to account for the laser beams offset at larger heights. After this, the predesigned motion pattern that encodes the start/stop signal for the silver dispenser and the pick-and-place mechanism are added to the drawing. Finally, all lines are color-coded to ensure proper fabrication order. All line-manipulations are summarized in Table 1.

Adding lines for soldering and pick-and-place trajectories: The original design file does not contain lines for picking up components from the component storage on the laser cutter's periphery and placing them onto the device geometry. We thus generate additional lines to guide the laser head between the pick-up locations of each component, and the target locations on the device as specified in the design file. To generate the lines, we use a simple pathplanning procedure that allows for obstacle-avoidance of all other

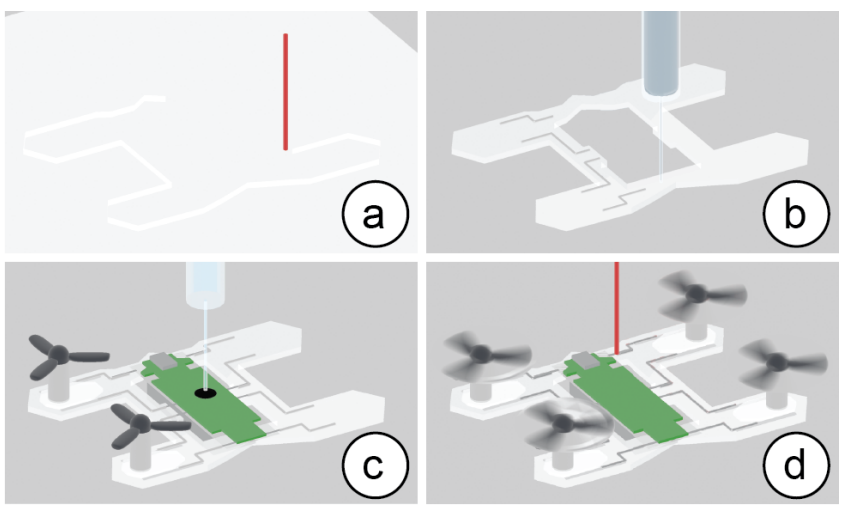

Figure 9: Visualization showing the resulting 3D geometry and order of fabrication steps: (a) cutting, (b) silver deposition, (c) component placement, (d) soldering and deployment.

components on the canvas. While the user only draws circuit traces once, the path of each circuit trace must be tracked twice: once for dispensing the silver with the silver dispenser and once for curing with the defocused laser. We thus create a second copy of the circuit traces to be used for curing. Finally, we append the motion patterns to signal to the silver dispenser and pick-and-place mechanism when to start/stop their operation as explained in section 6 .

Offsetting lines in the $X$-Y plane: As part of the post-processing, we apply offsets in the X-Y plane to the different fabrication steps. First the circuit traces and pick-and-place paths are offset to account for the physical offset between the laser head and the silver dispenser nozzle and the pick-and-place nozzle, respectively. Next, we apply a second round of offsets for curing and folding which involve the defocused laser at different heights. This is done to account for a slight misalignment between our laser and the z-axis, and requires a one-time calibration.

Order of execution: Most laser cutters, including the ULS.PLS.150D used here, order the execution of lines by color and allow assigning each color to a different power/speed/z-axis setting. To enforce the correct ordering of steps in our fabrication pipeline, we assign each step a designated color. Because lines of the same color are by default executed in the order in which they are drawn and written to the design file (.svg), we re-order lines of the same color, for instance, to encode which bend lines should be executed first in the event of serial folds. Our system automatically post-processes the design file with the modifications detailed above, producing a fabrication file ready for the laser cutter.

Loading onto the laser cutter: Following post-processing, users can load the output file into the regular laser cutter software to begin fabrication. Before starting fabrication, the user must also load the corresponding laser setting files (.las) that assigns power, speed, and z-axis settings for each color in the drawing. Settings for the pump and other hardware supporting the LaserFactory add-on's operation are programmed once onto its microcontroller and then valid independent of the design file. All laser cutter settings and hardware add-on settings are summarized in Table 1. Users place material in the bed, mount the LaserFactory add-on onto the laser 
head using three 3D printed clips, load components into the storage area, and then execute the job.

\section{APPLICATIONS}

The results from our evaluation indicate that devices created by Laserfactory can be used for applications requiring fine sensing and control, high frequency signals, and actuator driving with minimal loss. We demonstrate several of these capabilities through our example applications: a self-deploying quadcopter, a sensorequipped wristband, and a printed circuit board (Figure 10).

\subsection{Self-deploying quadcopter}

As our first example, we designed and fabricated a quadcopter (Figure 10a) to showcase three of LaserFactory's capabilities. When designing a quadcopter, one primary concern is minimizing its total mass. By using LaserFactory's ability to create geometry and circuit traces in tandem, we were able to reduce the area of the quadcopter platform to only contain material where circuit traces or components need to be placed, minimizing the overall mass. Second, this example illustrates LaserFactory's capability to fabricate devices that require non-trivial electrical currents, which is only practical with low resistance traces, such as those made by LaserFactory. Third, this example illustrates LaserFactory's capability to create devices end-to-end without human intervention; in this case the quadcopter can self-deploy directly from the build plate if needed. The total fabrication time of the quadcopter was 11 minutes. The electronic components placed as part of the fabrication process include the four rotors plus an integrated PCB and battery. Note that unlike regular SMD components that can be used as-is, we harvested these components from an existing quadcopter and therefore had to expose and position the pins by fastening them to acrylic substrates prior to placing them on the LaserFactory component storage area in order to be compatible with our technique.

\subsection{Personalized devices and wearables}

We fabricated a personalized, sensor-equipped wristband (Figure $10 \mathrm{~b}$ ) capable of sensing gestures that can be transmitted to control a mobile phone via bluetooth. It is comprised of a white sheet of acrylic with a personalized cutting pattern and a living hinge, in addition to an Arduino Sense BLE, battery, and passive components. By gesturing in one of four different directions (forward, backward, left, right), a different command is issued, and an LED changes color. The total fabrication time for this device was 9 minutes, after which the wristband can be picked out of the laser cutter and used immediately. This highlight's LaserFactory's ability to rapidly create personalized electronics on-demand. We also created capacitive sensors from traces themselves, showing how simple touch and humidity sensors as well as antennae can be fabricated without the need to procure dedicated external components.

\subsection{Printed circuit boards}

We fabricated an $\mathrm{H}$ bridge motor driver as an individual PCB (Figure 10c). $\mathrm{H}$ bridges are circuits used to switch the polarity of a load, allowing them to directly drive a DC motor bidirectionally. They are frequently used by hobbyists and electronic novices because microcontrollers, such as the Arduino platform, cannot drive motors

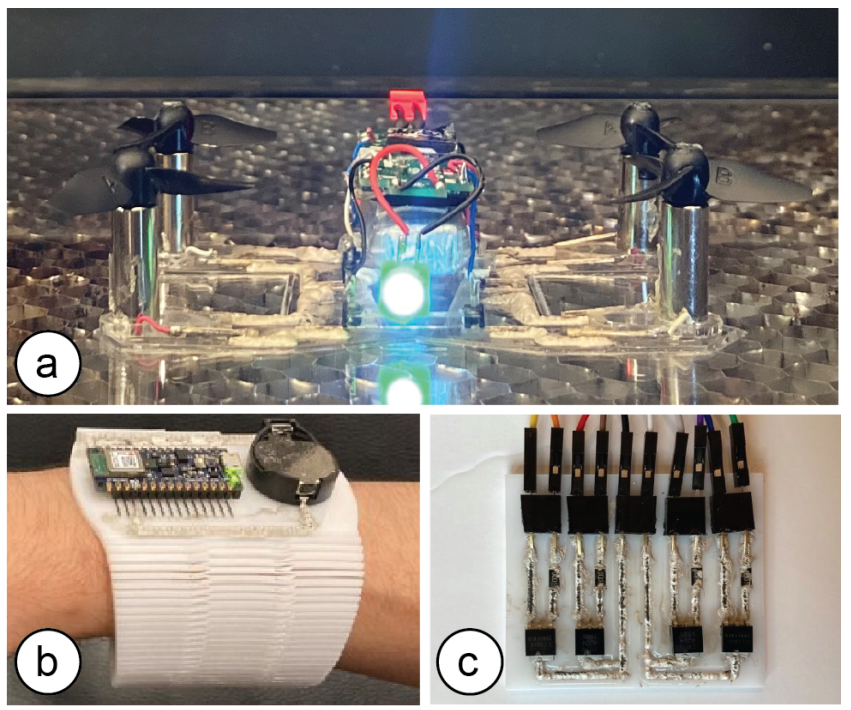

Figure 10: Functional Devices made with LaserFactory: (a) a quadcopter that can lift of the fabrication platform, (b) a sensor-equipped wristband capable of gesture recognition, and (c) a printed circuit board, specifically an $\mathrm{H}$ bridge.

directly from their pins. The $\mathrm{H}$ bridge takes 12 minutes to fabricate and is made up of 13 components: two PNP transistors, two NPN transistors, four resistors and five female header connectors. These connectors are used to easily mate with the four required control signals and a motor. This application demonstrates LaserFactory's capability to create ICs from basic SMD components, such as transistors and resistors. Since transistors and resistors can be used to create a variety of different ICs with different functionality (e.g., $\mathrm{H}$ bridges, Op Amps), this example showcases that LaserFactory can create a diverse set of devices even when only basic components are loaded into the component storage area. Thus, rather than ordering the ICs, LaserFactory can make them on demand. Moreover, as a single fabrication file encodes all fabrication instructions, such files can be easily shared and downloaded in order to fabricate PCBs that other experts have designed.

\section{LIMITATIONS AND FUTURE WORK}

We next discuss design decisions made while developing the LaserFactory system and lay out future opportunities for improvements.

Transferability between different laser cutters: Just as power, speed and height settings must be characterized for cutting the same material on two different laser cutters, the same is true for the settings of the LaserFactory add-on. The laser cutter settings in Table 1,3D printed clips for mounting the add-on, and the motion signal used are all calibrated for use on our specific laser cutter machine, i.e. the ULS.PLS.150D system. Thus, the settings need to be re-calibrated to work with the specific laser power, laser head shape, and carriage motion that is expected to differ between machines. Underlying this is the requirement for the firmware to allow reading an SVG/PDF file line by line, and to not automatically smooth all accelerations. For laser soldering, we tested and confirmed its compatibility with a range of power, speed and height permutations. 
This suggests that laser cutters within a range of power ratings could potentially be compatible for use with laser soldering as long as they have an adjustable platform height. While outside the scope of our work, it would be an important future work to validate this cross-device functioning.

Unidirectional communication: The platform agnosticism gained by leveraging uni-directional communications comes with the tradeoff that LaserFactory is not set up to transmit instructions back to the laser cutter firmware. In its current state, this prevents LaserFactory from being able to communicate interrupts to the laser cutter software to allow for potential interventions. Such interrupts would be useful to allow aborting fabrication in cases of malfunction or an unexpected contact detected by the pick-and-place sensor. One method of enabling bidirectional communication would be to fit the hardware add-on with an onboard transceiver, however interfacing it with laser cutter firmware would necessarily be platform specific.

Component Loading: In the current version of LaserFactory, we use a pre-loaded component storage inside the laser cutter that users can re-stock as needed. The storage consists of engraved footprints of electronic components that help the user align them correctly during loading. LaserFactory uses these positions to compute paths for the pick and place operations. The current system thus relies on foreknowledge about what components will be used. For future work, we plan to investigate how we can enable pre-stocking of a larger number of components and how to feed in components on-demand to accommodate real-time design decisions. In addition, LaserFactory's hardware currently does not support electronic component rotation. Thus, the orientation of pre-stocked components must match that of their virtual counterparts. For future work, we plan to add a rotation axis to the pick-and-place tool to extend the degrees of freedom available to designers. We also plan to investigate stocking material sheet in the component storage area, which can be cut and stacked to form multimaterial structures.

On-Demand Device Programming: The microcontrollers that are picked-and-placed as part of the fabrication process are currently manually pre-programmed ahead of time, requiring foreknowledge of the device in which they will be used and its context. For future work, we plan to explore methods for flashing programs on-demand to accommodate more versatile deployment; a promising candidate for this could be programming via infrared receivers (Kilobots [18]).

\section{CONCLUSION}

In this paper, we presented LaserFactory, an integrated fabrication platform that can rapidly create the geometry of a device, create its circuit traces and assemble components without manual intervention. We demonstrated how we can augment an existing laser cutter with a hardware add-on without interfacing with its underlying firmware by using a motion-based signaling technique that can inform the add-on when to start and stop its operation. We illustrated the two main features of our hardware add-on, a silver dispenser used for circuit trace creation and a pick-and-place mechanism used for assembling electronic components, and showed that the add-on can create high-resolution traces of high conductivity and assemble a range of different electronic components. We then showcased laser soldering, a technique that uses a $\mathrm{CO}_{2}$ laser to cure dispensed silver paste and discussed which laser cutter settings are most suitable to cure the traces. Finally, we showed our end-to-end design and fabrication pipeline consisting of a design tool, a visualization tool, and a post-processing script that transforms the design file into machine instructions for LaserFactory. We also showed example applications that included a quadcopter with actuators, a sensor-enhanced wristband, and a PCB assembled from basic transistors and resistors. For future work, we plan to evaluate the transferability of our add-on to different laser cutting machines, investigate how to improve component loading, and evaluate different methods to deploy control logic onto a fabricated device. Researchers have in recent decades made significant progress toward the long-term vision of being able to download a device file and have it fabricated at the push of a button. While laypeople can today do so for passive, primarily decorative objects via commercially available laser cutters and $3 \mathrm{D}$ printers, we hope that the fabrication of fully functional, electromechanical devices demonstrated in this paper will help spur further research toward that shared vision.

\section{ACKNOWLEDGMENTS}

This material is based upon work supported by the National Science Foundation under Grant No. 1716413. The work was also supported by a Microsoft Research Faculty Fellowship and The Royal Swedish Academy of Sciences.

\section{REFERENCES}

[1] Enkeleda Balliu, Henrik Andersson, Magnus Engholm, Thomas Öhlund, HansErik Nilsson, and Håkan Olin. 2018. Selective laser sintering of inkjet-printed silver nanoparticle inks on paper substrates to achieve highly conductive patterns. Scientific reports 8, 1 (2018), 1-9.

[2] Jesse Burstyn, Nicholas Fellion, Paul Strohmeier, and Roel Vertegaal. 2015. Printput: Resistive and capacitive input widgets for interactive 3D prints. In IFIP Conference on Human-Computer Interaction. Springer, Springer International Publishing, Bamberg, Germany, 332-339.

[3] Kentaro Fukuchi, Kazuhiro Jo, Akifumi Tomiyama, and Shunsuke Takao. 2012. Laser Cooking: A Novel Culinary Technique for Dry Heating Using a Laser Cutter and Vision Technology. In Proceedings of the ACM Multimedia 2012 Workshop on Multimedia for Cooking and Eating Activities (Nara, Japan) (CEA '12). Association for Computing Machinery, New York, NY, USA, 55-58. https://doi.org/10.1145/ 2390776.2390788

[4] Wei Gao, Yunbo Zhang, Diogo C. Nazzetta, Karthik Ramani, and Raymond J. Cipra. 2015. RevoMaker: Enabling Multi-Directional and Functionally-Embedded 3D Printing Using a Rotational Cuboidal Platform. In Proceedings of the 28th Annual ACM Symposium on User Interface Software \& Technology (Charlotte, NC, USA) (UIST '15). Association for Computing Machinery, New York, NY, USA, 437-446. https://doi.org/10.1145/2807442.2807476

[5] Daniel Groeger and Jürgen Steimle. 2019. LASEC: Instant Fabrication of Stretchable Circuits Using a Laser Cutter. In Proceedings of the 2019 CHI Conference on Human Factors in Computing Systems (Glasgow, Scotland Uk) (CHI '19). Association for Computing Machinery, New York, NY, USA, 1-14. https: //doi.org/10.1145/3290605.3300929

[6] Steve Hodges, Nicolas Villar, Nicholas Chen, Tushar Chugh, Jie Qi, Diana Nowacka, and Yoshihiro Kawahara. 2014. Circuit Stickers: Peel-and-Stick Construction of Interactive Electronic Prototypes. In Proceedings of the SIGCHI Conference on Human Factors in Computing Systems (Toronto, Ontario, Canada) (CHI '14). Association for Computing Machinery, New York, NY, USA, 1743-1746. https://doi.org/10.1145/2556288.2557150

[7] Shohei Katakura, Yuto Kuroki, and Keita Watanabe. 2019. A 3D Printer Head as a Robotic Manipulator. In Proceedings of the 32nd Annual ACM Symposium on User Interface Software and Technology (New Orleans, LA, USA) (UIST '19). Association for Computing Machinery, New York, NY, USA, 535-548. https: //doi.org/10.1145/3332165.3347885

[8] Yoshihiro Kawahara, Steve Hodges, Benjamin S. Cook, Cheng Zhang, and Gregory D. Abowd. 2013. Instant Inkjet Circuits: Lab-Based Inkjet Printing to Support Rapid Prototyping of UbiComp Devices. In Proceedings of the 2013 ACM International foint Conference on Pervasive and Ubiquitous Computing (Zurich, 
Switzerland) (UbiComp '13). Association for Computing Machinery, New York NY, USA, 363-372. https://doi.org/10.1145/2493432.2493486

[9] Yoshihiro Kawahara, Steve Hodges, Nan-Wei Gong, Simon Olberding, and Jürgen Steimle. 2014. Building functional prototypes using conductive inkjet printing. IEEE Pervasive computing 13, 3 (2014), 30-38.

[10] Mannu Lambrichts, Jose Maria Tijerina, Tom De Weyer, and Raf Ramakers 2020. DIY Fabrication of High Performance Multi-Layered Flexible PCBs. In Proceedings of the Fourteenth International Conference on Tangible, Embedded, and Embodied Interaction (Sydney NSW, Australia) (TEI '20). Association for Computing Machinery, New York, NY, USA, 565-571. https://doi.org/10.1145/ 3374920.3374988

[11] Jennifer A Lewis and Bok Y Ahn. 2015. Device fabrication: Three-dimensional printed electronics. Nature 518, 7537 (2015), 42-43.

[12] Joanne Lo and Eric Paulos. 2014. ShrinkyCircuits: Sketching, Shrinking, and Formgiving for Electronic Circuits. In Proceedings of the 27th Annual ACM Symposium on User Interface Software and Technology (Honolulu, Hawaii, USA) (UIST '14). Association for Computing Machinery, New York, NY, USA, 291-299. https://doi.org/10.1145/2642918.2647421

[13] David A. Mellis, Sam Jacoby, Leah Buechley, Hannah Perner-Wilson, and Jie Qi 2013. Microcontrollers as Material: Crafting Circuits with Paper, Conductive Ink, Electronic Components, and an "Untoolkit". In Proceedings of the 7th International Conference on Tangible, Embedded and Embodied Interaction (Barcelona, Spain) (TEI '13). Association for Computing Machinery, New York, NY, USA, 83-90. https://doi.org/10.1145/2460625.2460638

[14] Stefanie Mueller, Martin Fritzsche, Jan Kossmann, Maximilian Schneider Jonathan Striebel, and Patrick Baudisch. 2015. Scotty: Relocating Physical Objects Across Distances Using Destructive Scanning, Encryption, and 3D Printing. In Proceedings of the Ninth International Conference on Tangible, Embedded, and Embodied Interaction (Stanford, California, USA) (TEI '15). Association for Computing Machinery, New York, NY, USA, 233-240. https://doi.org/10.1145/2677199. 2680547

[15] Stefanie Mueller, Bastian Kruck, and Patrick Baudisch. 2013. LaserOrigami: Laser-Cutting 3D Objects. In Proceedings of the SIGCHI Conference on Human Factors in Computing Systems (Paris, France) (CHI '13). Association for Computing Machinery, New York, NY, USA, 2585-2592. https://doi.org/10.1145/2470654 2481358

[16] Martin EW Nisser, Samuel M Felton, Michael T Tolley, Michael Rubenstein, and Robert J Wood. 2016. Feedback-controlled self-folding of autonomous robot collectives. In 2016 IEEE/RS7 International Conference on Intelligent Robots and Systems (IROS) (Daejeon, South Korea). IEEE, IEEE, Washington, DC, USA, 12541261.

[17] Huaishu Peng, François Guimbretière, James McCann, and Scott Hudson. 2016 A 3D Printer for Interactive Electromagnetic Devices. In Proceedings of the 29th Annual Symposium on User Interface Software and Technology (Tokyo, Japan) (UIST '16). Association for Computing Machinery, New York, NY, USA, 553-562. https://doi.org/10.1145/2984511.2984523

[18] Michael Rubenstein, Christian Ahler, and Radhika Nagpal. 2012. Kilobot: A low cost scalable robot system for collective behaviors. In 2012 IEEE International Conference on Robotics and Automation. IEEE, IEEE, Saint Paul, MN, USA, 32933298.

[19] Daniel Saakes, Thomas Cambazard, Jun Mitani, and Takeo Igarashi. 2013. PacCAM: Material Capture and Interactive 2D Packing for Efficient Material Usage on CNC Cutting Machines. In Proceedings of the 26th Annual ACM Symposium on User Interface Software and Technology (St. Andrews, Scotland, United Kingdom) (UIST '13). Association for Computing Machinery, New York, NY, USA, 441-446. https://doi.org/10.1145/2501988.2501990

[20] Valkyrie Savage, Ryan Schmidt, Tovi Grossman, George Fitzmaurice, and Björn Hartmann. 2014. A Series of Tubes: Adding Interactivity to 3D Prints Using Internal Pipes. In Proceedings of the 27th Annual ACM Symposium on User Interface Software and Technology (Honolulu, Hawaii, USA) (UIST '14). Association for Computing Machinery, New York, NY, USA, 3-12. https://doi.org/10.1145/ 2642918.2647374

[21] Valkyrie Savage, Xiaohan Zhang, and Björn Hartmann. 2012. Midas: Fabricating Custom Capacitive Touch Sensors to Prototype Interactive Objects. In Proceedings of the 25th Annual ACM Symposium on User Interface Software and Technology (Cambridge, Massachusetts, USA) (UIST '12). Association for Computing Machinery, New York, NY, USA, 579-588. https://doi.org/10.1145/2380116.2380189

[22] Martin Schmitz, Mohammadreza Khalilbeigi, Matthias Balwierz, Roman Lissermann, Max Mühlhäuser, and Jürgen Steimle. 2015. Capricate: A Fabrication
Pipeline to Design and 3D Print Capacitive Touch Sensors for Interactive Objects. In Proceedings of the 28th Annual ACM Symposium on User Interface Software \& Technology (Charlotte, NC, USA) (UIST '15). Association for Computing Machinery, New York, NY, USA, 253-258. https://doi.org/10.1145/2807442.2807503

[23] Martin Schmitz, Jürgen Steimle, Jochen Huber, Niloofar Dezfuli, and Max Mühlhäuser. 2017. Flexibles: Deformation-Aware 3D-Printed Tangibles for Capacitive Touchscreens. In Proceedings of the $2017 \mathrm{CHI}$ Conference on $\mathrm{Hu}$ man Factors in Computing Systems (Denver, Colorado, USA) (CHI '17). Association for Computing Machinery, New York, NY, USA, 1001-1014. https: //doi.org/10.1145/3025453.3025663

[24] Martin Schmitz, Martin Stitz, Florian Müller, Markus Funk, and Max Mühlhäuser. 2019. ../Trilaterate: A Fabrication Pipeline to Design and 3D Print Hover-, Touch-, and Force-Sensitive Objects. In Proceedings of the 2019 CHI Conference on Human Factors in Computing Systems (Glasgow, Scotland Uk) (CHI '19). Association for Computing Machinery, New York, NY, USA, 1-13. https://doi.org/10.1145/ 3290605.3300684

[25] Saiganesh Swaminathan, Kadri Bugra Ozutemiz, Carmel Majidi, and Scott E. Hudson. 2019. FiberWire: Embedding Electronic Function into 3D Printed Mechanically Strong, Lightweight Carbon Fiber Composite Objects. In Proceedings of the 2019 CHI Conference on Human Factors in Computing Systems (Glasgow, Scotland Uk) (CHI '19). Association for Computing Machinery, New York, NY, USA, 1-11. https://doi.org/10.1145/3290605.3300797

[26] Alexander Teibrich, Stefanie Mueller, François Guimbretière, Robert Kovacs, Stefan Neubert, and Patrick Baudisch. 2015. Patching Physical Objects. In Proceedings of the 28th Annual ACM Symposium on User Interface Software \& Technology (Charlotte, NC, USA) (UIST '15). Association for Computing Machinery, New York, NY, USA, 83-91. https://doi.org/10.1145/2807442.2807467

[27] Udayan Umapathi, Hsiang-Ting Chen, Stefanie Mueller, Ludwig Wall, Anna Seufert, and Patrick Baudisch. 2015. LaserStacker: Fabricating 3D Objects by Laser Cutting and Welding. In Proceedings of the 28th Annual ACM Symposium on User Interface Software \& Technology (Charlotte, NC, USA) (UIST '15). Association for Computing Machinery, New York, NY, USA, 575-582. https://doi.org/10. $1145 / 2807442.2807512$

[28] Nobuyuki Umetani and Ryan Schmidt. 2017. SurfCuit: Surface-Mounted Circuits on 3D Prints. IEEE Comput. Graph. Appl. 38, 3 (May 2017), 52-60. https://doi. org/10.1109/MCG.2017.40

[29] Alexander D Valentine, Travis A Busbee, John William Boley, Jordan R Raney, Alex Chortos, Arda Kotikian, John Daniel Berrigan, Michael F Durstock, and Jennifer A Lewis. 2017. Hybrid 3D printing of soft electronics. advanced Materials 29, 40 (2017), 1703817.

[30] Joshua Vasquez, Hannah Twigg-Smith, Jasper Tran O'Leary, and Nadya Peek. 2020. Jubilee: An Extensible Machine for Multi-Tool Fabrication. In Proceedings of the 2020 CHI Conference on Human Factors in Computing Systems (Honolulu, HI, USA) (CHI '20). Association for Computing Machinery, New York, NY, USA, 1-13. https://doi.org/10.1145/3313831.3376425

[31] Voxel8. 2019. Voxel8. Voxel8. Retrieved November 21, 2019 from https://www. voxel8.com/

[32] Guanyun Wang, Lining Yao, Wen Wang, Jifei Ou, Chin-Yi Cheng, and Hiroshi Ishii. 2016. XPrint: A Modularized Liquid Printer for Smart Materials Deposition. In Proceedings of the 2016 CHI Conference on Human Factors in Computing Systems (San Jose, California, USA) (CHI '16). Association for Computing Machinery, New York, NY, USA, 5743-5752. https://doi.org/10.1145/2858036.2858281

[33] Christian Weichel, John Hardy, Jason Alexander, and Hans Gellersen. 2015. ReForm: Integrating Physical and Digital Design through Bidirectional Fabrication. In Proceedings of the 28th Annual ACM Symposium on User Interface Software \& Technology (Charlotte, NC, USA) (UIST '15). Association for Computing Machinery, New York, NY, USA, 93-102. https://doi.org/10.1145/2807442.2807451

[34] Junichi Yamaoka, Mustafa Doga Dogan, Katarina Bulovic, Kazuya Saito, Yoshihiro Kawahara, Yasuaki Kakehi, and Stefanie Mueller. 2019. FoldTronics: Creating 3D Objects with Integrated Electronics Using Foldable Honeycomb Structures. In Proceedings of the 2019 CHI Conference on Human Factors in Computing Systems (Glasgow, Scotland Uk) (CHI '19). Association for Computing Machinery, New York, NY, USA, 1-14. https://doi.org/10.1145/3290605.3300858

[35] Junyi Zhu, Lotta-Gili Blumberg, Yunyi Zhu, Martin Nisser, Ethan Levi Carlson, Xin Wen, Kevin Shum, Jessica Ayeley Quaye, and Stefanie Mueller. 2020. CurveBoards: Integrating Breadboards into Physical Objects to Prototype Function in the Context of Form. In Proceedings of the 2020 CHI Conference on Human Factors in Computing Systems (Honolulu, HI, USA) (CHI '20). Association for Computing Machinery, New York, NY, USA, 1-13. https://doi.org/10.1145/3313831.3376617 